\title{
Interference-Based Optical Measurement of Fluidic Flow in a Hollow-Core Fiber
}

\author{
Min-Hwan LEE, Sung-Hyun KIM, Eun-Sun KIM, and In-Kag HWANG* \\ Department of Physics, Chonnam National University, 33, Yongbong-ro, Buk-gu, Gwangju, 61186, Republic of Korea \\ *Corresponding author: In-Kag HWANG ～E-mail: ikhwang@jnu.ac.kr
}

\begin{abstract}
In this study, we present speed and displacement measurements of micro-fluid in a hollow-core optical fiber, where an optical interference signal is created by two guided beams reflected at a fixed facet and a moving fluid end. By counting the number of intensity oscillations of the signal, the movement of the fluid end is successfully traced with high accuracy. Furthermore, we could detect the change in curvature diameters of the fluid end depending on the flow direction by monitoring the visibility of the interference signal.
\end{abstract}

Keywords: Fiber optic sensing; micro channel fluidic flow; fluidic velocimetry; optical fiber interferometry

Citation: Min-Hwan LEE, Sung-Hyun KIM, Eun-Sun KIM, and In-Kag HWANG, "Interference-Based Optical Measurement of Fluidic Flow in a Hollow-Core Fiber," Photonic Sensors, 2018, 8(1): 7-12.

\section{Introduction}

The measurement of fluid speed is an important technique widely required in many areas including industry [1], medicine [2], biology [3], and microfluidics. Among the various methods developed so far, non-invasive measurements using ultrasonic [4] or optical waves have been widely used for a flow in closed tubes that do not allow for a mechanical access of the fluid. When the fluid includes particles or bubbles to scatter optical or acoustic waves, the fluid speed can be measured using its transit time for a given distance or the amount of Doppler shift in the reflected wave [1]. Ultrasonic transit time flow meters measure the difference in the transit time of ultrasonic pulses propagating in and opposite the flow direction [5].

In this study, we report speed and displacement measurements of fluidic flow using optical interference. The interference occurs between the probe beam reflected at the moving fluidic end and the reference beam reflected at a fixed point. The number of oscillations in the interference signal corresponds to the distance that the fluid travels. This approach provides high accuracy at the optical wavelength [6] and does not require any scatterer in the fluid for speed measurement provided that the fluid end forms a well-defined boundary. One important requirement of this scheme is that the capillary or tube for the fluid flow should provide optical guidance with a tolerable loss. In our study, we use the hollow-core photonic bandgap fiber (HC-PBF) instead of a conventional capillary tube for low-loss delivery of the optical signal. The fiber has a hollow tube with a diameter of $20 \mu \mathrm{m}$ that guides both fluid and light. The tube is surrounded by a honey-comb photonic crystal structure preventing optical leakage at the $1.55-\mu \mathrm{m}$ wavelength band [7].

Received: 21 June 2017 / Revised: 26 September 2017

(C) The Author(s) 2017. This article is published with open access at Springerlink.com

DOI: $10.1007 / \mathrm{s} 13320-017-0447-8$

Article type: Regular 


\section{Experimental setup}

The experimental setup is shown in Fig. 1. The 1-m-long HC-PBF (hollow core photonic band-gap fiber) is connected to a single-mode fiber by butt-coupling and a capillary tube with a hole-diameter of $20 \mu \mathrm{m}$ by fusion-splice. Mercury is chosen as the fluid because of its low viscosity and high optical reflectivity [8]. A distributed-feedback (DFB) laser with a wavelength of $1550 \mathrm{~nm}$ is used as the optical source. The laser light is divided into two light beams after propagating through the circulator from Port 1 to Port 2. One of the light beams is reflected at the end of the single-mode fiber by Fresnel reflection [9], and the other beam is reflected at the end of mercury after propagating in the HC-PBF. The interference signal of the two beams is detected at Port 3 . We use a micro syringe pump (Harvard Bioscience Co. PY2 70-2209) to control the position of the mercury end [10]. (a)

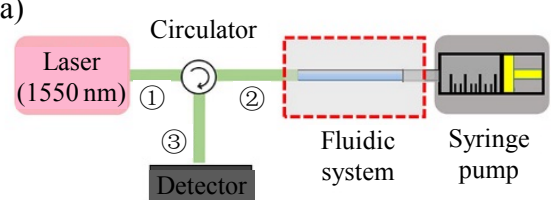

(b)

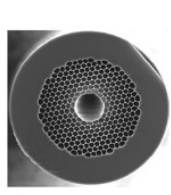

(c)

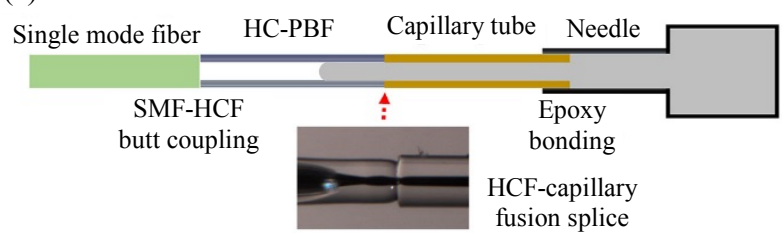

Fig. 1 Schematic diagram of the system: (a) experimental setup, (b) cross-section of the HC-PBF used in the experiment, and (c) schematic of the optical fiber-capillary-syringe pump connection.

The intensity of the interference beam is given as

$$
\begin{gathered}
\left|E_{1}\right|=E_{0} \sqrt{R_{1}},\left|E_{2}\right|=E_{0} T_{1} \sqrt{R_{2}} \\
I_{\text {tot }}=\left|E_{1}\right|^{2}+\left|E_{2}\right|^{2}+2\left|E_{1}\right|\left|E_{2}\right| \cos \delta,\left(\delta=2 \pi \frac{2 \Delta l}{\lambda}\right)
\end{gathered}
$$

where $\Delta l$ is the distance between the end of the single-mode fiber and the end of the fluid, as shown in Fig. 2. Here, we assume that $E_{2}$ has the same polarization as $E_{1}$, and the effective refractive index of the fundamental mode in $\mathrm{HC}-\mathrm{PBF}$ is 1 . One cycle of the fluctuation in the interference signal corresponds to a fluid movement of $\Delta l=\lambda / 2=775 \mathrm{~nm}$.

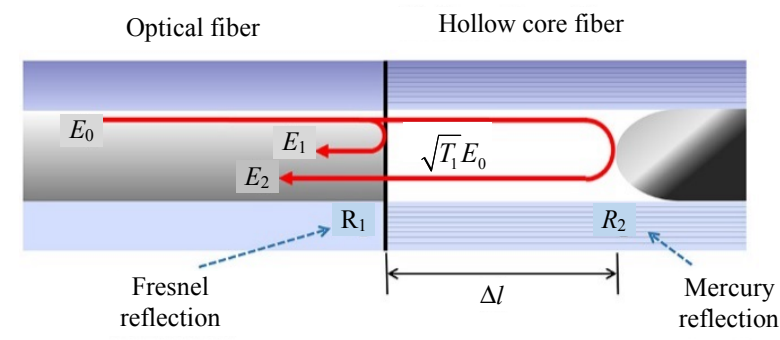

Fig. 2 Interference of two optical beams reflected at the facet of the single-mode fiber $\left(E_{1}\right)$ and fluid end $\left(E_{2}\right)$.

The observed waveform of $I_{\text {tot }}$ for $300 \mathrm{~s}$ is shown in Fig. 3(a). The slow-varying waveform between $0 \mathrm{~s}$ and $100 \mathrm{~s}$ indicates the slow movement of fluid. Figure 3(b) shows the magnified waveform at $120 \mathrm{~s}$, where signal fluctuation is caused by the interference of $E_{1}$ and $E_{2}$. The single-pass coupling loss of single mode fiber (SMF) to $\mathrm{HC}-\mathrm{PCF}$ is $3.5 \mathrm{~dB}$, and the reflection loss at the mercury end is $7 \mathrm{~dB}-10 \mathrm{~dB}$, resulting in the total insertion loss of $14 \mathrm{~dB}-17 \mathrm{~dB}$.

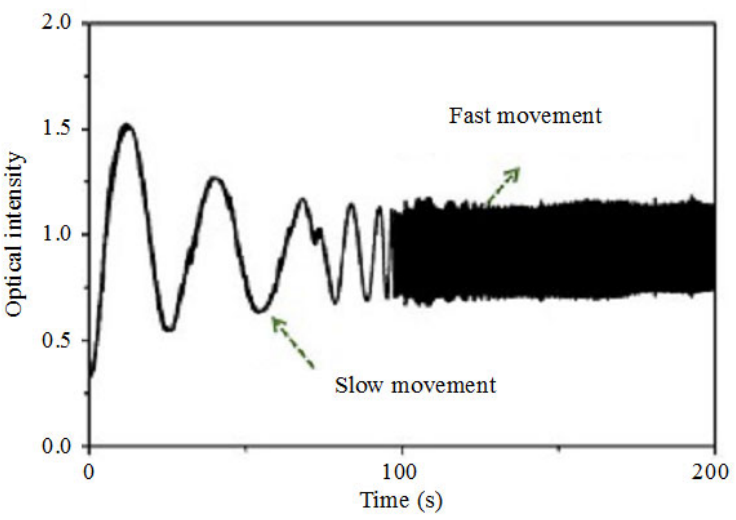

(a)

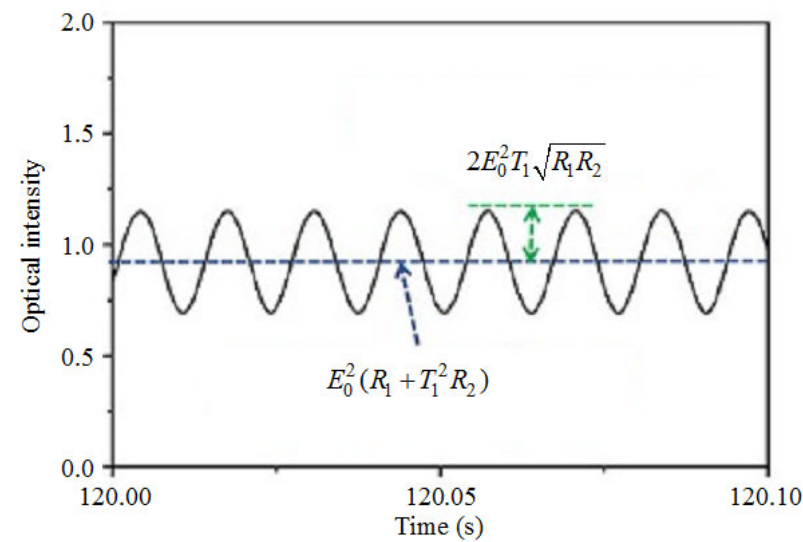

(b)

Fig. 3 Interference signals: (a) detected optical signal with the fluid movement and (b) the corresponding magnified waveform. 


\section{Analysis of fluid movement}

By counting the number of oscillations, we could obtain the information about the position of the fluid end, as shown in Fig. 4(a). Figure 4(b) shows the velocity of the fluid, which is calculated by taking the time derivative of the position. Continuous injection of $100 \mathrm{nl}$ mercury at a speed of $100 \mathrm{nl} / \mathrm{min}$ for $80 \mathrm{~s}$ is followed by a suction of the same amount after an intermission of about $80 \mathrm{~s}$. The fluid velocity during the injection is much faster and immediately responsive to the pumping. This happens because the injection pressure is applied directly by the syringe pump (the maximum pressure of $175 \mathrm{psi}$ ) while the suction relies solely on the atmospheric pressure (14.7psi). The maximum fluid speeds are $4.3 \mathrm{~mm} / \mathrm{s}$ and $1.0 \mathrm{~mm} / \mathrm{s}$ during the injection and suction, respectively. The total travel distance of the fluid is about $200 \mathrm{~mm}$. After the injection and suction of the same amount, the final position of the fluid is close to the starting point with an error of a few millimeters, indicating no significant fluid leakage in the system.

The accuracy of the position measurement will be approximately a half of the laser wavelength in the ideal case. However, the interference signal can be affected not only by the fluid movement but also by the unstable laser wavelength or variation of the fiber length ( $\Delta l$ in Fig. 2) caused by mechanical vibration or temperature change of the fiber. Such a noise fluctuation of the signal should grow proportional to the fiber length $\Delta l$. We find that the signal is fluctuating at a rate of a few cycles per second for $\Delta l=1 \mathrm{~m}$ when the fluid is not flowing, indicating the signal noise is induced by the aforementioned reasons. This amount of noise fluctuation contributes an error of a few micrometers per second to the fluid velocity measurement. Therefore, the error of the position measurement grows with the measurement duration. If a measurement is performed for $100 \mathrm{~s}$, the error in the fluid position will be a few hundred micrometers.
Note that this error is relatively small compared with that of a conventional fiber optic interferometry using single-mode fibers, where the optical path length $\Delta l$ varies significantly because of the strong temperature dependence of the core refractive index [11]. The use of a hollow-core fiber results in the reduced temperature sensitivity.

The maximum range of the fluid movement measurable with the current setup is determined by the linewidth of the laser because the interference fringes disappear when the round-trip path length of $2 \Delta l$ is longer than the coherence length of the laser. For the DFB laser with a bandwidth of $1.0 \mathrm{MHz}$, the maximum range of the measurement is $110 \mathrm{~m}$.
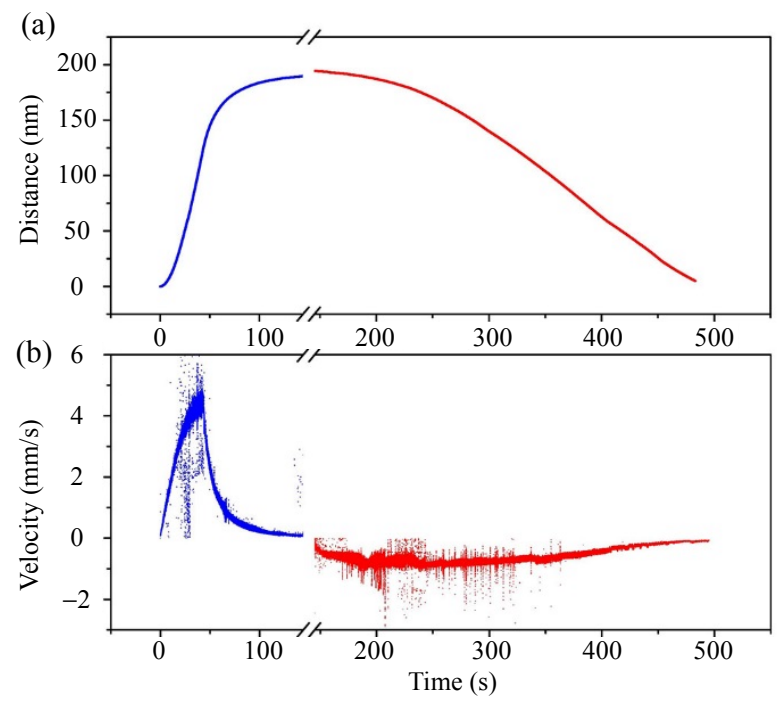

Fig. 4 Analysis of fluid movement based on the optical signals: (a) travel distance of the fluid obtained from the optical interference signal and (b) velocity of the fluid obtained by taking a time derivative of the distance data.

\section{Changes in curvature of fluid end}

Figure 5 shows the interference signal observed at the transition between the injection and suction modes. Note that there is a large difference in the interference visibility for the two modes, as well as periodic beating in the signal. The origin of the beating is the inference between spatial modes in the probe beam. The loss of the higher-order spatial modes is not large enough to consider the HC-PBF as a pure single-mode fiber in the short length of the 
fiber. The magnitude of the beating could be diminished when the higher-order modes are suppressed by use of a longer length of HC-PBF. Now let us focus on the visibility changes after switching the flow direction. The best explanation for this is that the optical reflectivity $\left(R_{2}\right)$ is at the mercury end, and thus, the amplitude of $\left|E_{2}\right|$ changes between the injection and suction modes. $R_{2}$ is dependent on the curvature of the end surface of the fluid. Because of surface tension, the fluid tends to form a spherical surface at the fluid end. However, when the fluid moves to the empty region in the injection mode, the advance of the fluid occurs near the central axis of the HC-PBF while the fluid will have zero velocity at the boundary with no slipping [12]. This fluid dynamics results in a more convex surface with a greater curvature. On the other hand, in the suction mode, the curvature decreases, and a flatter surface is formed at the fluid end. Such a behavior is observed everywhere in the whole length of HC-PBF depending only on the moving direction of mercury. Based on the experimental data, the optical reflectivity $\left(R_{2}\right)$ of mercury is calculated to be $18 \%$ and $11 \%$ for the suction and injection modes, respectively.

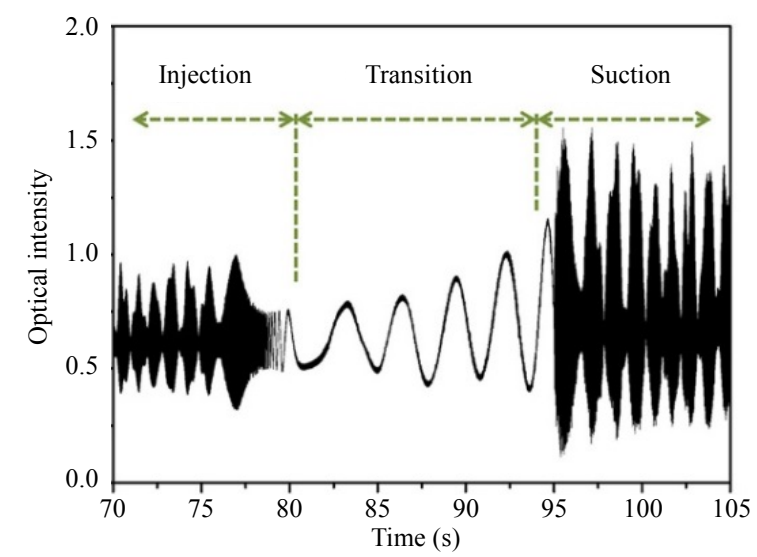

Fig. 5 Variation of the interferometric visibility of signal for the transition between the injection and suction modes.

Here we try to estimate the radius of curvature of the mercury end resulting in the relatively low optical reflectance mentioned above. In the scalar wave approximation, the optical reflectance $\left(R_{2}\right)$ in a waveguide is determined by the overlap integral

[13]:

$$
R_{2}\left|\int_{0}^{\infty} \psi_{1} \psi_{2}^{*} d r\right|^{2}
$$

where

$$
\begin{gathered}
\psi_{1}=\sqrt{\frac{2}{\pi}} \frac{1}{a} \mathrm{e}^{-\frac{r^{2}}{a^{2}}} \\
\psi_{2}= \begin{cases}\sqrt{\frac{2}{\pi}} \frac{1}{a} \mathrm{e}^{-\frac{r^{2}}{a^{2}}+\Delta \Delta \Phi(r)}, & , 0<r<\frac{d}{2} \\
0 & , r>\frac{d}{2}\end{cases} \\
\Delta \Phi(r)=\frac{4 \pi\left(r_{c}-\sqrt{r_{c}^{2}-r^{2}}\right)}{\lambda}
\end{gathered}
$$

where $\psi_{1}$ is the Gaussian field of the fundamental mode, $\psi_{2}$ is the electric field of the optical wave reflected at the curved surface of fluid with a curvature radius of $r_{c}$ [Fig. 6(a)], and $\Delta \Phi(r)$ is the phase shift corresponding to the travel distance of the reflected wave dependent on the radial position. The travel distance is the shortest at the center of the tube $(r=0)$ and the longest at the tube wall $(r=d / 2)$.

In Figs. 6(b) and 6(c), we plot field distributions of real parts of $\psi_{1}$ and $\psi_{2}$, respectively. Black circles denote the fiber core. The phase of $\psi_{2}$ is significantly distorted due to the small radius of curvature of $50 \mu \mathrm{m}$ and also cut at the core boundary. The numerical result for the optical reflectivity $\left(R_{2}\right)$ is plotted as a function of $r_{c}$ in Fig. 7. Compared with the experimental results for the optical reflectance of 0.11 and 0.18 in injection and suction modes, respectively, we estimate the radius of curvature of mercury end to be $67 \mu \mathrm{m}$ and $82 \mu \mathrm{m}$ in injection and suction modes, respectively.

(a)

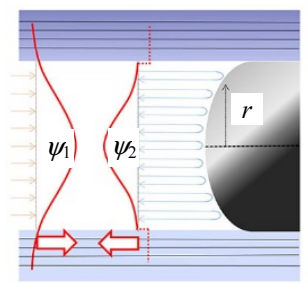

(b)

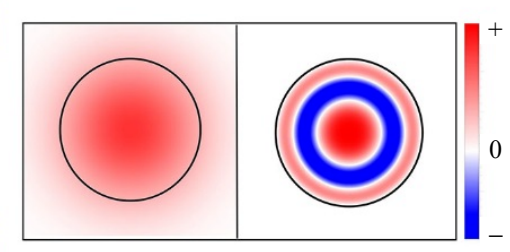

Fig. 6 Mode profiles of incident and reflected beams: (a) fundamental guided mode $\psi_{1}$ and reflected wave $\psi_{2}$, electric field profiles of (b) $\psi_{1}$ and (c) $\psi_{2}$ (for $r_{c}=50 \mu \mathrm{m}$ ). 


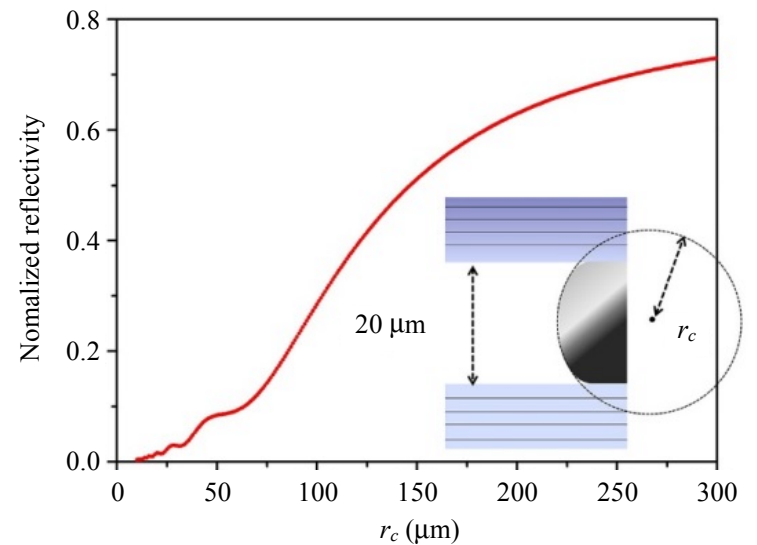

Fig. 7 Optical reflectivity as a function of the radius of curvature.

\section{Conclusions}

We demonstrate the position and speed monitoring of liquid in a hollow-core fiber by using optical interference. The interference signal is generated by two guided beams reflected at the fiber facet and liquid end. The number of intensity oscillations and visibility variation of the interference signal are acquired to retrieve the information about the liquid position and optical reflectivity at the liquid end, at the same time. We find that the change in the optical reflectivity is caused by the change in the curvature of the liquid surface during the transition between the injection and suction modes. This information can be effectively used to determine the flow direction while the flow speed is measured by fringe counting of the interference signal.

Our approach is useful for analyzing fluid flow with high accuracy in a microfluidic channel. There are two conditions to be met in order to apply this method. Firstly, the system requires a well-defined reflective point such liquid-air or liquid-liquid interface. Secondly, the fluid channel should also provide optical guidance of the probe beam with a tolerable loss. For example, hollow-core photonic bandgap fibers providing single-mode guidance of optical wave are currently available with the tube diameters of $5 \mu \mathrm{m}-20 \mu \mathrm{m}$. The two conditions are readily met in applications of level sensors [14] and
Venturi flow meter [15]. In a Venturi flow meter, HC-PCF is installed at the side of a pipe as a probe to measure the pressure of the fluid which is dependent on the flow rate in the pipe. The small diameter $(\sim 100 \mu \mathrm{m})$ of the probe is advantageous for access to microfluidic channel or blood vessel. Our sensor is also applicable to various optofluidic devices [16], where micro control of a liquid drop is required.

\section{Acknowledgment}

This study was financially supported by Chonnam National University (2016).

Open Access This article is distributed under the terms of the Creative Commons Attribution 4.0 International License (http://creativecommons.org/ licenses/by/4.0/), which permits unrestricted use, distribution, and reproduction in any medium, provided you give appropriate credit to the original author(s) and the source, provide a link to the Creative Commons license, and indicate if changes were made.

\section{References}

[1] A. Zaouk, E. Salvetat, J. Sakaya, F. Maury, and G. Constant, "Various chemical mechanisms for the crystal growth of III-V semiconductors using coordination compounds as starting material in the MOCVD process," Journal of Crystal Growth, 1981, 55(1): 135-144.

[2] G. Kaltsas and A. G. Nassiopoulou, "Gas flow meter for application in medical equipment for respiratory control: study of the housing," Sensors \& Actuators A: Physical, 2004, 110(1-3): 413-422.

[3] G. E. Nilsson, T. Tenland, and P. A. Oberg, "Evaluation of a laser doppler flowmeter for measurement of tissue blood flow," IEEE Transactions on Biomedical Engineering, 1980, 27(10): 597-604.

[4] M. Dell'Isola, M. Cannizzo, and M. Diritti, "Measurement of high-pressure natural gas flow using ultrasonic flowmeters," Measurment, 1997, 20(2): 75-89.

[5] D. L. Franklin, D. W. Baker, R. M. Ellis, and R. F. Rushmer, "A pulsed ultrasonic flowmeter," Ire Transactions on Medical Electronics, 1959, 6(4): 204-206.

[6] W. T. Estler, "High-accuracy displacement interferometry refin air," Applied Optics, 1985, 24(6): 808-815. 
[7] V. Dangui, M. J. F. Digonnet, and G. S. Kino, "Modeling of the propagation loss and backscattering in air-core photonic-bandgap fibers," Journal of Lightwave Technology, 2009, 27(17): 3783-3789.

[8] W. E. Mueller, "Reflectivity of liquid mercury," Journal of the Optical Society of America, 1969, 59(9): 1246-1247.

[9] F. Couny, F. Benabid, and P. S. Light, "Reduction of fresnel back-reflection at splice interface between hollow core PCF and single-mode fiber," IEEE Photonics Technology Letters, 2007, 19(13): 1020-1022.

[10] M. H. Lee, S. H. Kim, E. S. Kim, J. T. Kim, and I. K. Hwang, "Analyses of micro-fluid flow in a hollow core fiber based on optical interference," in Proceedings of the IEEE Photonics Conference, Burlingame, California, USA, 2012, pp. 943-944.

[11] W. H. Hatton and M. Nishimura, "Temperature dependence of chromatic dispersion in single mode fibers," Journal of Lightwave Technology, 1986, 4(10): 1552-1555.

[12] S. Richardson, "On the no-slip boundary condition," Journal of Fluid Mechanics, 1973, 59(4): 707-719.

[13] A. Ghatak and K. Thyagarajan, Introduction to fiber optics. Cambridge, England: Cambridge University Press, 1998: 151-155.

[14] E. Musayev and S. E. Karlik, "A novel liquid level detection method and its implementation," Sensors and Actuators A: Physical, 2003, 109(1-2): 21-24.

[15] W. Z. Song and D. Psaltis, "Imaging based optofluidic air flow meter with polymer interferometers defined by soft lithography," Optics Express, 2010, 18(16): 16561-16566.

[16] D. Psaltis, S. R. Quake, and C. Yang, "Developing optofluidic technology through the fusion of microfluidics and optics," Nature, 2006, 442: 381-386. 